2. O’Connell JB, Maggard MA, Manunga J, Tomlinson JS, Reber HA, Ko CY et al. Survival after resection of ampullary carcinoma: a national population-based study. Ann Surg Oncol. 2008;15:1820-7.

3. Temesgen WM, Wachtel M, Dissanaike S. Osteoclasticgiant cell tumor of the pancreas. Int J Surg Case Rep. 2014;5:175-9.

4. Shishido-Hara Y, Kurata A, Fujiwara M, Itoh H, Imoto S, Kamma H. Two cases of breast carcinoma with osteoclastic giant cells: Are the osteoclastic giant cells pro-tumoural differentiation of macrophages? Diagn Pathol. 2010;5:55.

5. Matsuzawa G, Shirabe K, Gion T, Tsujita E, Ooya M, Kajiyama K. Surgically resected undifferentiated carcinoma with osteoclast-like giant cells of the periampullary region involving the orifice of the papilla of Vater: Report of a case. Surg Today. 2010;40:376-9.

6. Fischer HP, Zhou H. Pathogenesis of carcinoma of the papilla of Vater. J Hepatobiliary Pancreat Surg. 2004;11:301-9.

7. Westgaard A, Tafjord S, Farstad IN, Cvancarova M, Eide TJ, Mathison $\mathrm{O}$ et al. Pancreatobiliary versus intestinal histologic type of differentiation is an independent prognostic factor in resected periampullary adenocarcinoma. BMC Cancer. 2008;8:170-4.

8. Moore JC, Bentz JS, Hilden K, Adler DG. Osteoclastic and pleomorphic giant cell tumors of the pancreas: a review of clinical, endoscopic, and pathologic features. World J Gastrointest Endosc. 2010;2:15-9.

\section{Median arcuate ligament syndrome (MALS) in rural Prakasam district of Andhra Pradesh}

Harjola $^{1}$ was the first surgeon who described the celiac axis compression syndrome or Median Arcuate Ligament syndrome in 1963. Subsequently, J David Dunbar and Samuel Marable ${ }^{2}$ also spoke about the same in 1965 and so, it is called the Harjola-Marable syndrome. The median arcuate ligament is formed near the 12th thoracic vertebra and usually comes into contact with the aorta above the branch point of the celiac artery. ${ }^{3}$ Sometimes, the median arcuate ligament passes in front of the celiac artery, thus compressing it and presents with the triad of abdominal pain, weight loss and vomiting.

One theory proposes that compression of the celiac artery causes is chemiaor decreased blood flow to the abdominal organs, thus leading to pain. Another hypothesis that there is compression not only of the celiac artery but also of the celiac ganglia. ${ }^{4}$ It typically occurs in young patients (20 to 40 years of age) and is more common in thin women. ${ }^{5}$ We report one such case which was diagnosed using CT angiography and was managed effectively using a holistic approach by a team of doctors.

\section{Case Report}

A 21-year old unmarried rural young male had consulted in multiple hospitals for postprandial pain. After a threeyear history of intermittent abdominal pain and decreased appetite, he presented to us with recurrent episodes of epigastric pain of moderate to severe intensity associated with vomiting and constipation. He had been suffering similar complaints at least 5 to 6 times in a year with each episode is lasting for 5 to 7 days and responding to supportive treatment. The frequency had increased in the last one year. He is the second sibling of a consanguineous family. No significant health problems were noticed in his family members. He is neither an alcoholic nor a smoker and has lost $8 \mathrm{kgs}$ in 2 year. There is no history of neurological symptoms, skin manifestations, urinary symptoms and per rectal bleeding. Per abdomen was soft with mild tenderness observed in the epigastric region.

Complete blood count (CBC), amylase, lipase, liver function tests, renal function tests, fasting blood sugar, bleeding time and clotting time were normal. This patient was negative for HBsAg, HCV and HIV infections. Peripheral blood smear for malarial parasite, Widal test for Salmonella and serum levels of electrolytes like sodium, potassium and chloride were also normal.

Ultrasound abdomen and X-ray barium did not reveal any significant problem. Gastritis was noticed on upper GI endoscopy while colonoscopy was normal. 
Magnetic Resonance Cholangio Pancreaticography (MRCP) was normal while CT angiography revealed severe stenosis of the celiac trunk secondary to extrinsic compression by the median arcuate ligament connecting the diaphragmatic crura giving a hooked appearance to the celiac trunk (Figure 1).

The patient was taken up for laparoscopic dissection to release the compressing median arcuate ligament and its impingement on the celiac artery. The fibrous attachments around the aorta and the origin of the celiac artery were carefully dissected and divided. The division of these fibres was carried out circumferentially until the artery appeared to be free of any external compression. The patient tolerated this maneuver well and was kept under observation for a few days. A few days later he developed features of ileus due to hypokalemia and significantly improved with supportive treatment. A post-operative CT angiography revealed a normal celiac trunk with no evidence of stenosis. His condition was good at the time of follow-up after few weeks of surgery and his episodic epigastric pain had not reappeared (Figure 2).

The patient's GI symptoms have not recurred after surgery for the last three months and he has been able to continue with his usual diet without any significant problems or difficulties in performing his daily activities (Figure 3).

\section{Discussion}

The median arcuate ligament is a fibrous arch that unites the diaphragmatic crura crossing the aorta superior to the celiac axis at the level of the first lumbar vertebra.The incidence of this syndrome is reported to be between 1.76 to $4 \%{ }^{6}$

While diagnosing these patients, other causes of visceral pain should be excluded such as biliary pain and pain from ulcer disease. CT angiography is the gold standard in the diagnosis of this disease as it can detect focal narrowing of the celiac axis, particularly in sagittal sections. This narrowing has a characteristic hooked appearance which was also seen in our patient's CTangiography

The treatment of MAL syndrome is aimed at restoring normal blood flow in the celiac axis and surgical

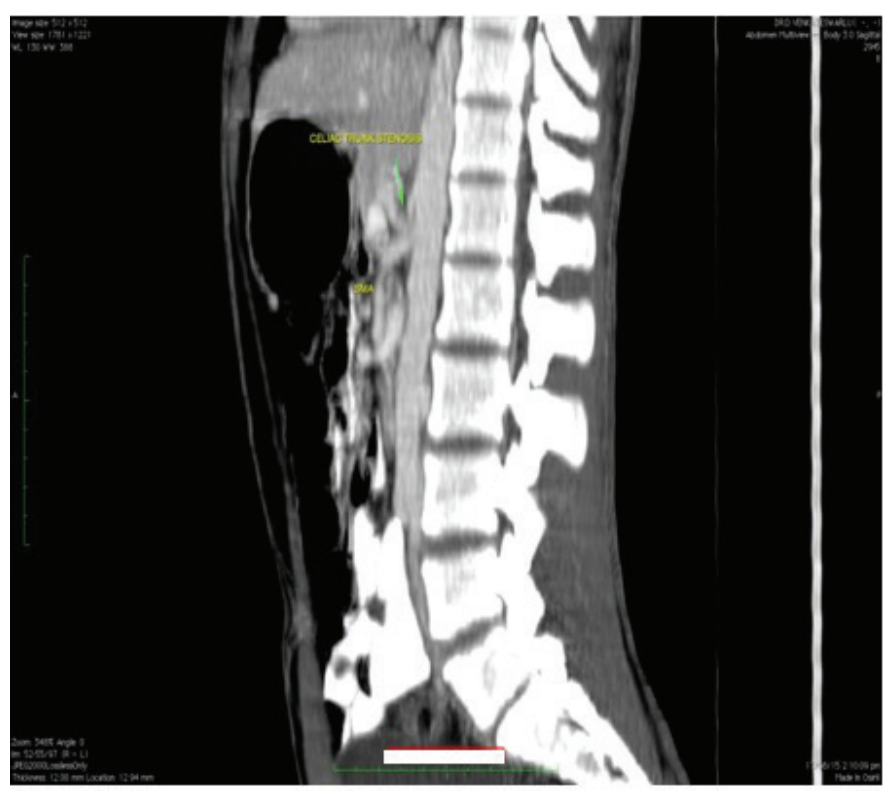

Figure 1: Sagittal view of the computed tomography angiography showing compression of the celiac artery and celiac trunk stenosis.

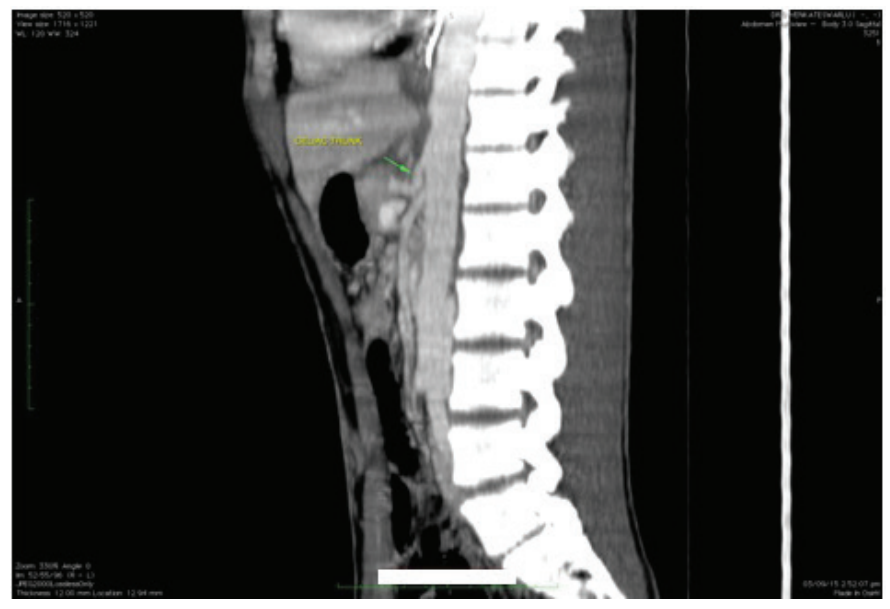

Figure 2: Sagittal view of the computed tomography angiography showing released celiac trunk stenosis.

decompression of the celiac axis in our patient resulted in the resolution of abdominal pain and a return to a full diet within a few days. Even though several treatment options have been described for MALS, the management classically involves the surgical division of the MAL fibers. 


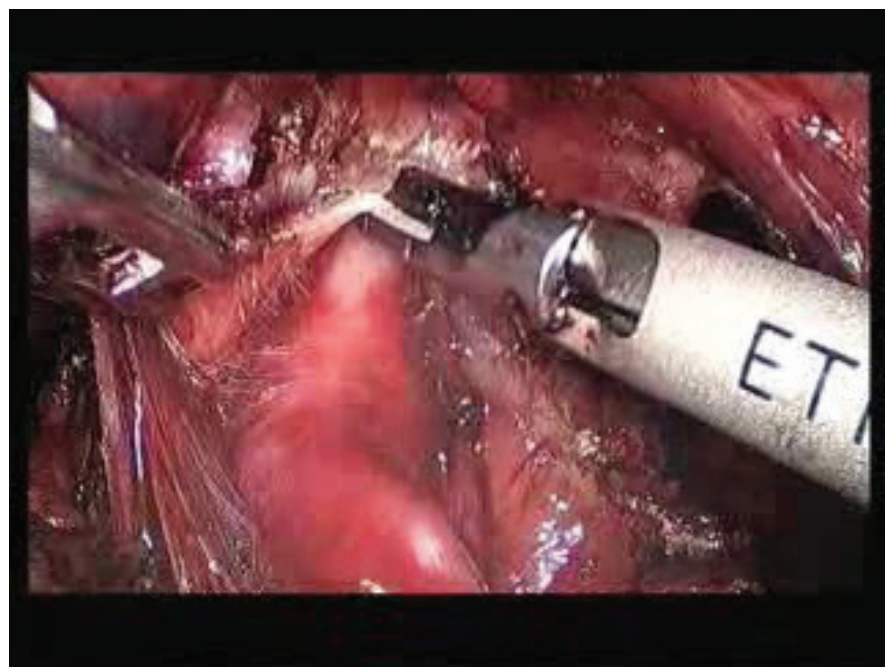

Figure 3: Laparoscopic Separation of Median Arcuate Ligament.

To conclude, definitive angiographic findings, along with clinical impression, are needed to reach the confirmative diagnosis of MALS. The laparoscopic dissection of the MAL from below upward is more beneficial than the same procedure from above downward since in the latter case, the aorta is at risk of injury. We recommend a multidisciplinary team with a holistic approach to care for patients with this complex and rare disease.

\section{VENKATESWARLU ${ }^{1}$ B CHANDRA SEKHAR ${ }^{2}$ CH JAYA KISHORE ${ }^{3}$ BT RAO ${ }^{4}$}

${ }^{1}$ Department of Gastroenterology, Vijaya Sree Hospitals Gastro and Liver Care Centre, Ongole, Andhra Pradesh, India.

${ }^{2}$ Department of Surgical Gastroenterology, Sunrise Hospitals, Vijayawada, Andhra Pradesh, India.

${ }^{3}$ Radiologist, Sai Vijaya Diagnostic Centre, Ongole, Andhra Pradesh, India.

${ }^{4}$ Department of Community Medicine, Rajiv Gandhi Institute of Medical Sciences, Ongole, Andhra Pradesh, India.

Correspondence: D Venkateswarlu Email:devarakonda2013@gmail.com

\section{References}

1. Harjola PT. A Rare Obstruction of the Coeliac Artery. Report of a Case. Ann Chir Gynaecol Fenn. 1963;52:54750.

2. Dunbar JD, Molnar W, Beman FF, Marable SA. Compression of the celiac trunk and abdominal angina. Am J Roentgenol Radium Ther Nucl Med. 1965;95(3):731-44.

3. Horton KM, Talamini MA, Fishman EK. Median arcuate ligament syndrome: evaluation with CT angiography. Radiographics. 2005;25:1177-82.

4. Duncan AA. Median arcuate ligament syndrome. Curr Treat Options Cardiovasc Med. 10:112-6.

5. Sproat IA, Pozniak MA, Kennell TW. US case of the day: median arcuate ligament syndrome.Radio Graphics. 1993;13:1400-2.

6. Göya C, Hamidi C, Hattapoğlu S, Çetinçakmak MG, Teke M, Kuday S. Diagnosis of median arcuate ligament syndrome on multidetector computed tomography. J Med Cases. 2013;4:616-9.

\section{An enigmatic right iliac fossa mass}

Eosinophilic colitis (EC) is an extremely rare disease in the spectrum of Primary Eosinophilic Gastrointestinal Disease (EGID), which also includes Eosinophilic Esophagitis (EE), Eosinophilic Gastritis (EG) and Eosinophilic Gastroenteritis. In all the above, there is significant tissue eosinophilia causing chronic inflammation. However, the common causes of tissue eosinophilia (parasitic infestation, drug reaction and malignancy) must be ruled out first. ${ }^{1}$ The disease can affect the entire gastrointestinal tract and its etiology is stillunclear. Bimodal age distribution is observed, affecting infants and young adults. Chronic diarrhea leading to malabsorption and significant weight loss is the classical presentation while intestinal obstruction is very rare. 